\title{
Recombinant VP60 in the form of virion-like particles as a potential vaccine against rabbit hemorrhagic disease virus ${ }^{\star}$
}

\author{
Beata Gromadzka1凶, Bogusław Szewczyk ${ }^{1}$, Grażyna Konopa ${ }^{2}$, Andrzej Fitzner ${ }^{3}$ and \\ Andrzej Kęsy ${ }^{3}$ \\ ${ }^{1}$ Department of Molecular Virology, Intercollegiate Faculty of Biotechnology, University of Gdansk and Medical \\ University of Gdańsk, Gdańsk, Poland; ${ }^{2}$ Department of Molecular Biology, University of Gdańsk, Gdańsk, Po- \\ land; ${ }^{3}$ Department of Foot-and-Mouth Disease, National Veterinary Research Institute, Zduńska Wola, Poland; \\ 凶e-mail: g.beata@biotech.univ.gda.pl
}

Received: 11 January, 2006; revised: 09 May, 2006; accepted: 10 May, 2006

available on-line: 30 May, 2006

\begin{abstract}
Rabbit hemorrhagic disease virus (RHDV) which causes a highly contagious disease of wild and domestic rabbits belongs to the family Caliciviridae. It is a small, positive single-stranded RNA virus with a genome of $7.5 \mathrm{~kb}$ and has a diameter of approximately $40 \mathrm{~nm}$. In negatively stained electron micrographs the virus shows typical calicivirus morphology with regularly arranged cupshaped structures on the surface. It is a major pathogen of rabbits in many countries. Vp60 - a coat protein of molecular mass around $60 \mathrm{kDa}$ is the major antigen of RHDV. It is present as 90 dimeric units per virion particle. We have expressed VP60 gene in the baculovirus system with the aim to use it as a potential vaccine against RHDV and a diagnostic reagent in immunological tests. cDNA of the $v p 60$ gene of strain SGM, was cloned into a baculovirus transfer vector as full-length gene, as well as truncated gene lacking $6005^{\prime}$-terminal nucleotides. The sequence of SGM VP60 differed markedly from that of the reference strain. Full-length recombinant VP60 protein from the SGM strain self-assembled to form virus-like particles (VLPs). These particles observed by electron microscopy were morphologically similar to native virions and were able to agglutinate human group 0 erythrocytes. After immunization the recombinant particles induced RHDV-specific antibodies in rabbits and guinea pigs. Rabbits immunized with the VLPs were fully protected against challenge with a virulent RHDV.
\end{abstract}

Keywords: RHDV, VP60, baculovirus, VLP

\section{INTRODUCTION}

Rabbit hemorrhagic disease is caused in most cases by a hemagglutinating virus belonging to the family Caliciviridae, genus Lagovirus, which so far has not been propagated in vitro.

RHDV is the causative agent of a contagious disease of wild and domestic rabbit, Oryctolagus cuniculus. Infected animals show typical pathological lesions, including necrotic hepatitis, hemorrhaging and edema of lungs and trachea, with a 60-100\% mortality rate occurring 24-48 h after infection (Liu et al., 1984; Ohlinger et al., 1990).
RHDV is a non-enveloped RNA virus with a diameter of approx. 36-40 nm. The virons are composed of a positive single-stranded RNA, $7.5 \mathrm{~kb}$ in length and a single major capsid protein with a molecular mass of $60-70 \mathrm{kDa}$. In addition to the genomic RNA, RHDV produces a subgenomic RNA of $2.2 \mathrm{~kb}$ that covers one third of the genome from the 3 ' end and probably plays an important role in expression of the capsid protein (Meyers, et al., 1991; Gall et al., 1992; Nagesha et al., 1995; Martinez-Torrecuadrada et al., 1998).

Sequence analysis of the RHDV genome revealed an open reading frame (ORF1) that en-

^This work was presented at the BioMillenium Conference in Gdańsk, October 2005, Poland.

Abbreviations: AcNPV, Autographa californica nuclear polyhedrosis virus; BCIP, 5-bromo-4-chloro-3-indolyl-phosphate; EM, electron microscopy; HI, hemagglutination inhibition tests; IPMA, immunoperoxidase monolayer assay; NBT, 4-nitro blue tetrazolium chloride; PBS, phosphate-buffered saline; RHDV, rabbit hemorrhagic disease virus; TM, Tris/magnesium buffer; VLP, virus-like particles; VP60, viral coat protein $60 \mathrm{kDa}$. 
codes a polyprotein of $257 \mathrm{kDa}$. The $3^{\prime}$ region of the RHDV genome contains a second open reading frame (ORF2) of 351 nucleotides that overlaps ORF1 by several nucleotides and encodes a polypeptide of $12.7 \mathrm{kDa}$ which has been described as structural protein VP10.

Sequence comparison has allowed for the identification of non-structural and structural proteins on the large polyprotein. The non-structural proteins identified were as follows: RNA helicase, protease, and RNA-dependent polymerase, homologous to the $2 \mathrm{C}$ and $3 \mathrm{C}$ and $3 \mathrm{D}$ proteins of picornaviruses, respectively.

Cryo-electron microscopy studies on RHDV show that the structural protein folds into two main domains which are held together by third domain that acts as a hinge region (Cappuci et al., 1998).

It is postulated that the capsid of RHDV is formed by two functionally different concentric shells: the inner shell of the N-terminal half of the VP60 protein, called S domain, contains and protects the genome, and the outer shell comprising the Cterminal half of the structural protein (P2 domains), contains two highly variable regions designated $\mathrm{C}$ and $\mathrm{E}$, and also contains the main antigenic region (Cappuci et al., 1991; 1995; Meyers et al., 1991; Sibilia et al., 1995).

The aim of our project was to construct a potential vaccine against rabbit hemorrhagic disease. This paper describes the expression of the structural capsid protein gene vp60, from a Polish strain SGM in the baculovirus system. The recombinant protein self-assembled to form virus-like particles which were able to agglutinate human group 0 erythrocytes. Rabbits immunized with these particles were fully protected against a challenge with a virulent RHDV.

\section{MATERIALS AND METHODS}

Virus cDNA synthesis. The SGM strain with hemagglutinating activity was isolated in 1998 in Poland.

RNA was extracted with an RNA isolation kit (Qiagen), from a liver sample from experimentally infected rabbits. cDNA synthesis was performed as described (Fitzner et al., 1999).

PCR amplification. Most DNA manipulations were performed using standard methods (Sambrook et al., 1989).

PCR amplifications were carried out using primers designed from known sequences of the German reference strain of RHDV (M67473) and gave a product of $1796 \mathrm{bp}$ encoding full-length VP60 protein (VP1,VP3), and a product of $1196 \mathrm{bp}$ encoding a truncated form (600 bp from the 3' end) of vp60 gene (VP1,VP2). PCR conditions were as follows: $4 \mathrm{~min}$ at $94^{\circ} \mathrm{C}, 30 \times\left(1 \mathrm{~min}\right.$ at $94^{\circ} \mathrm{C}, 1 \mathrm{~min}$ at $55^{\circ} \mathrm{C}$, $2 \mathrm{~min}$ at $\left.72^{\circ} \mathrm{C}\right)$. The sequences of the primers were: VP1 (GCTAGTCGACGTTATGGAGGGCAAAGCCCR), VP2 (CGTCTCGAGCTGTGCACCTGAAGTGTTGGT), VP3 (GCCTCGAGGCACCTGCAAGTCCCAATCCG).

Plasmid construction and sequencing. The product of amplification was cloned into pGEM-Teasy vector as described in the pGEM-T easy manual (Promega). The plasmid was propagated in Escherichia coli cells and purified on an affinity column (A\&A Biotechnology).

DNA sequencing was performed using universal primers for T7 and Sp6 promoter in PGEM-T easy vector.

Construction of a recombinant baculovirus transfer vector and generation of baculovirus recombinants. Viral RNA was purified as described (Fitzner et al., 1999). After reverse transcription and amplification the cDNA was cloned into the pGEMT-Easy vector (Promega) and then subcloned into the baculovirus transfer vector pFastBac 1 (Invitrogen), by using the NotI and XhoI restriction enzyme sites. The recombinant baculovirus transfer constructs, pFastBac1-vp60-1 and pFastBac1-vp60-2, which contained full-length vp60 and truncated capsid gene were used for site-specific recombination in E. coli (strain DH10bac) (Parra \& Prieto, 1990), respectively. A recombinant bacmid containing the full or truncated form of the vp60 gene was then used to transfect insect cells (Sf9 cell line).

Recombinant baculoviruses containing both forms of the vp60 gene were identified by the IPMA and by PCR reaction with specific primers based on the vp60 sequence.

Production and purification of VP60 VLPs. The recombinant baculoviruses were used to infect Sf9 insect cells at a multiplicity of infection (MOI) of 1 or 0.1 and cell cultures were harvested $4-5$ days postinfection. The harvested cells and supernatant were separated by centrifugation in a microfuge at 13000 r.p.m. for $10 \mathrm{~min}$ at room temperature. Both the supernatant and the cell lysates were examined for recombinant VP60 capsids by SDS/PAGE and immunoblotting with hyperimmune rabbit serum against RHDV. Alkaline phosphatase conjugated to goat anti-rabbit immunoglobulin (Santa Cruz) was used as a secondary antibody, and NBT and BCIP as substrates for color development. The proteins in polyacrylamide gels were stained with Coomassie brilliant blue.

For purification of VP60 VLPs, Sf9 cells in suspension culture were infected with recombinant baculovirus at an MOI of 0.1 and harvested 5 days postinfection. Supernatant was collected after centrifugation in a microfuge at 13000 r.p.m. for 10 min. VP60 VLPs from the supernatant were purified 
by sucrose gradient (60-10\%) centrifugation at 80000 $\times g$ for $1.5 \mathrm{~h}$ at $4^{\circ} \mathrm{C}$ (SW $41 \mathrm{Ti}$ Beckman rotor). To determine the density and composition of the VP60 VLPs, $1 \mathrm{ml}$ fractions were collected from the sucrose gradients with thin pipette tips and were analyzed by SDS/PAGE and Western blotting. The purified VLPs were used as antigens in ELISA reaction experiments and to immunize rabbits and guinea pigs as described below.

Determination of the dynamics of recombinant protein production in Sf9 cells. Sf9 cells infected with recombinant baculovirus were harvested daily from 1 to 5 days post infection. The supernatants were separated from the cell pellet by centrifugation in a microfuge at 13000 r.p.m. for 10 min. The cell pellets were suspended in cell lysis buffer, and the recombinant proteins localized in the membrane fraction, cytoplasm and supernatant were detected by SDS/PAGE and immunoblotting. For immunoblotting, the VP60 VLPs in the supernantant were precipitated with $10 \%$ trichloroacetic acid; the resulting pellets were dissolved in PBS before application to a gel.

Production of hyperimmune antiserum in rabbits and guinea pigs. Two guinea pigs and two rabbits were immunized either with the recombinant protein purified from a PVDF membrane or with VP60 VLP mixed with Freund's complete adjuvant via subcutaneous injection followed by two booster injections, 2 weeks apart, of the same dose in Freund's incomplete adjuvant. The guinea pigs and rabbits were bled 2 weeks after the last booster injection. The serum was collected and stored at $-20^{\circ} \mathrm{C}$ until use. Specific anti-VLP sera had titer of $1: 20000$ in guinea pigs and in the range of 1:2500-1:10240 in rabbits.

Electron microscopy. The VP60 VLPs purified from the supernatant of infected Sf9 cells by sucrose gradient centrifugation were diluted in TM buffer (Tris and $\mathrm{MgCl}_{2}$ buffer). Particles were adsorbed onto carbon-coated grids, stained with $2 \%$ uranyl acetate, and examined immediately in a Philips CM 100 electron microscope.

Vaccination. Recombinant VP60 capsid protein expressed in the baculovirus S9 cell expression system which self assembled into virus-like particles - VLPs was used for vaccinations.

The RHD VLP titre from the Sf 9 medium determined by the HA test was $1: 10240$.

The animals. Seven seronegative adult rabbits (3 months old) were vaccinated with a dose of $1 \mathrm{ml}$ VLPs suspension, containing 150-200 $\mu \mathrm{g}$ protein. The VLPs were administered subcutaneously. A second group of four rabbits was maintained as controls. Two of them were non-immunized, and two were injected with Sf9 medium containing no recombinant baculovirus expression product.
The antibody response of the rabbits was determined by HI and ELISA 12 days post vaccination.

On the same day all animals were challenged by subcutaneous inoculation with a dose of $1 \mathrm{ml}$ of virulent RHDV exceeding one hundred times the $\mathrm{LD}_{50}$ (lethal dose, $50 \%$ ) and representing a HA titer of $1: 10240$.

\section{RESULTS AND DISCUSSION}

The baculovirus expression system has been used to express proteins of many DNA and RNA viruses. It has numerous advantages over other expression systems: high expression efficacy, eukaryotic posttranslational modifications, preservation of biological properties of recombinant proteins, and self-assembly of viral capsid proteins into VLPs for both DNA and RNA viruses (Jiang et al., 1992; Cappuci et al., 1998). For instance, the capsid proteins of Norwalk virus were expressed efficiently in baculovirus, and the recombinant molecules self-assembled into VLPs that were antigenically and morphologically similar to their respective native viruses of that family (Gall et al., 1992). In this study, we have expressed the vp60 gene of rabbit hemorrhagic disease virus in the baculovirus system with the aim to use it as a potential vaccine against RHDV and a diagnostic reagent in ELISA tests. cDNA of the vp60 gene of the Polish strain SGM was cloned into the baculovirus transfer vector as a full-length gene as well as a truncated gene lacking 600 3'-terminal nucleotides. Using the baculovirus system, rabbit hemorrhagic disease virus VP60 of Polish hemaglutinating isolate SGM was synthesized at high level.

\section{Expression of the VP60 capsid protein gene in insect cells}

After transfection of Sf9 cells with recombinant bacmid DNA encoding the full and truncated form of the vp60 gene, the recombinant baculoviruses were identified by the IPMA test (Fig. 1) and confirmed by PCR. The recombinant baculoviruses were amplified and the plaque assay was preformed to determine their titer. The recombinant viruses were used to infect insect cells and the conditions of infection were optimized to obtain the highest production of VP60. A major polypeptide band with a molecular mass of $60 \mathrm{kDa}$ was identified in cell lysates (membrane fraction and cytoplasm) and supernatant after analysis by SDS/PAGE (10\%) and staining with Coomasie brilliant blue. In Western blotting, the fulllength protein was reactive with a polyclonal rabbit serum against RHDV (Fig. 2). The full-length protein 


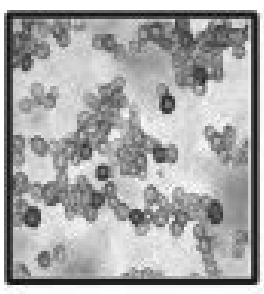

A

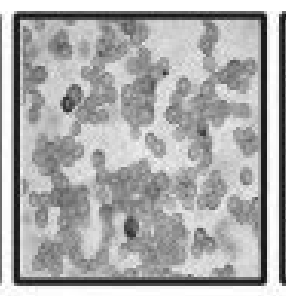

B

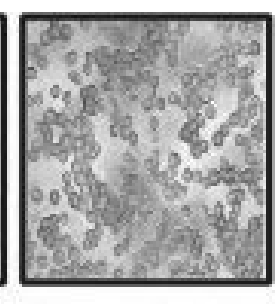

C
Figure 1. Immunodetection of recombinant VP60 protein in insect cells by immunoperoxidase monolayer assay.

The immunoperoxidase monolayer assay measures antigen-antibody interactions in virus-infected cells that are cultured in multiwell plates, and then sequentially reacted with specific antibody, horseradish peroxidase-labeled antiglobulin and precipitable chromogenic substrate. A) Baculovirus coding for full-length VP60; B) Baculovirus coding truncated VP60; C) Wild-type baculovirus. Rabbit polyclonal anti-RHDV sera were used in IPMA test.

band was clearly visible in the supernatant fractions, suggesting that the recombinant capsids were released into the medium of the infected insect cells. The truncated form of the VP60 protein was also identified in cell lysates, but not in the supernatant; the majority of it was found in the membrane fraction, with a molecular mass of $40 \mathrm{kDa}$. This protein was also reactive with the monospecific antisera.

Dynamics of the rVP60 capsid protein production in Sf9 cells

Sf9 cells infected with recombinant baculoviruses were harvested daily from 1 to 5 days post infection. Cell lysates and supernatants were examined either by SDS/PAGE and Western blotting or by ELISA. Our results indicated that the full-length protein was produced starting from the first day after infection and it was released into the medium. The peak of the rVP60 polypeptide production detected in the supernatant was from day 4 to day 5 post infection. The truncated form of the rVP60

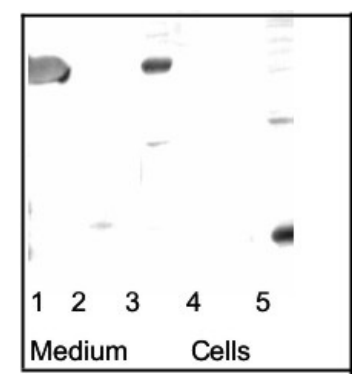

Figure 2. Western blot analysis of recombinant proteins VP60.

The medium and cells lysate were examined by SDS/ PAGE in reducing conditions $\left(1 \times 10^{6}\right.$ cells/lane). The Sf9 cells were collected $96 \mathrm{~h}$ post infection. Anti-RHDV serum (1:100 dilution) was used to detect the recombinant protein. Medium: 1, pFastBacSGM1800; 2, pFastBacSGM1200; 3, WT AcNPV; Cells: 4, pFastBacSGM1800; 5, FastBacSGM1200.

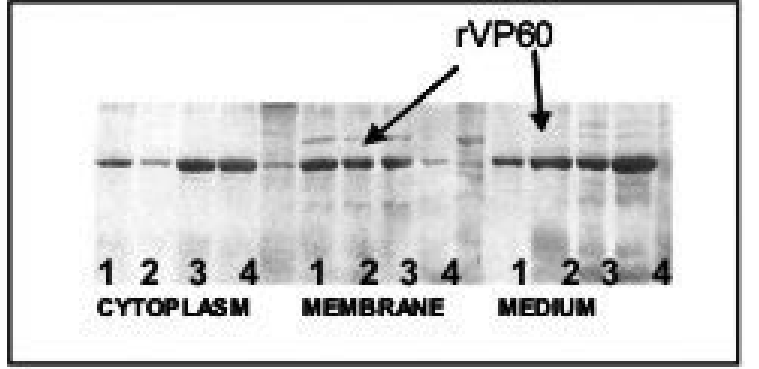

Figure 3. Dynamics of rVP60 protein production in Sf9 cells.

Cell membrane fraction, cytoplasm and medium were examined by SDS/PAGE in reducing conditions $\left(0.8 \times 10^{6}\right.$ cells/lane). The fractions were collected in 1, 2, 3 or 4 days post infection. Anti-RHDV serum (1:100 dilution) was used to detect the recombinant protein VP60. The figure shows full-length recombinant VP60.

protein was detected from the first day post infection, but the optimum time for harvest of the rVP60 polypeptide was also at day 4 to 5 post infection, as in the case of the full length protein. The localization of the truncated form of VP60 was different, and we detected the largest amount of this protein in the membrane fraction of the cell lysates (Fig. 3).

\section{Self-assembly of the rVP60 polypeptide into VLPs}

Because the full-length capsids composed of rVP60 were released into the medium, we purified the potential VLPs from the supernatant by sucrose gradient centrifugation. Immunobloting analysis of the sucrose gradient fraction indicated that a few of the fractions contained the recombinant capsids composed of a $60 \mathrm{kDa}$ polypeptide which reacted with polyclonal antisera and monoclonal antibodies confirming that the rVP60 capsids were antigenically similar to native virions. The VLPs were morphologically similar to native virons in size (average diameter 35-37 nm) and possessed characteristic cupshapped surface depressions (Fig. 4).

We also examined the membrane fraction containing the truncated form of the rVP60 protein. Purification of the potential VLPs from the mem-

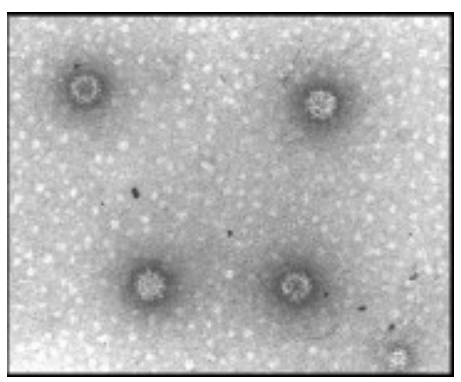

Figure 4. Electron microscopy of RHDV-VLP.

Sucrose gradient fraction was applied to carbon-coated grids, negatively stained with $2 \%$ uranyl acetate, and observed at a magnification of $\times 39000$ with a Philips CM 100 electron microscope. 


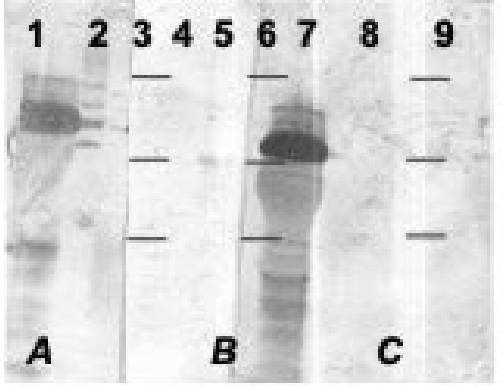

Figure 5. Western blot analysis of VP60 using polyclonal serum obtained after immunization of a rabbit with purified recombinant VP60 protein.

The rabbit was immunized with recombinant VP60 protein purified from PVDF membrane. First immunization was with $100 \mu \mathrm{g}$ of protein and the second after 2 weeks with $50 \mu \mathrm{g}$ of protein. A) anti-RHDV, B) serum 0, C) antirVP60; the polyclonal sera were used in a 1:100 dilution. 1, 4, 7, VP60; 2, 5, 8, WT AcNPV; 3, 6, 9, Protein MW marker.

brane fraction as well as the cytoplasm fraction on a sucrose gradient and further analysis of the gradient fractions by ELISA or immunobloting indicated that the truncated polypeptide did not form the VLP structure.

This result suggested that the 600 3'-terminal nucleotides are probably responsible for the aggregation of the capsid protein to form VLP. Antigenicities of the VLPs and purified RHDV were compared by Western blot analysis. Polyclonal sera and monoclonal antibodies recognized the same protein of $60 \mathrm{kDa}$, a molecular mass corresponding to the monomer form of the structural capsid protein rVP60, in the RHD virus and in VLP samples. The amount of the rVP60 protein in the cell lysates was lower at day 5 post infection than at day 2 post infection suggesting that the full-length capsid protein may have a hydrophobic character and is exported via the cellular membranes to the medium.

The truncated form of the capsid protein was also recognized by the anti-RHDV polyclonal serum and by the anti-VLP polyclonal serum. Under reducing conditions we observed three proteins of 30,25 , and $15 \mathrm{kDa}$, which may indicate that many forms of truncated rVP60 protein are formed or that it undergoes degradation. The protein was localized in the membrane fraction.

\section{Immunogenicity of the VP60 VLP}

Two guinea pigs and two rabbits were hyperimmunized with purified VP60 VLPs and bled 2 weeks after the last injection. The sera had high titers of antibodies against VP60 capsids when tested by ELISA (Fitzner A., Niedbalski W., unpublished). The guinea pig and rabbit sera also specifically recognized the rVP60 capsid protein (the full-length and truncated form of rVP60) in Western blotting
(Fig. 5). The sera tested by ELISA and HA test had titers up to $1: 20000$.

We also immunized guinea pigs and rabbis with full-length rVP60 protein purified from the PVDF membrane. The sera had a lower titer of antibodies against VP60 (1:5000) but also specifically recognized both forms of the VP60 protein and the VLPs.

\section{Vaccination}

Seven seronegative adult rabbits were vaccinated with a dose of $1 \mathrm{ml}$ VLPs suspension, containing 150-200 $\mu \mathrm{g}$ protein. After the challenge with RHDV, seven vaccinated rabbits survived and four non-immunized died between $48-72 \mathrm{~h}$ post infection showing the clinical signs of RHD. All vaccinated rabbits showed seroconversion and the anti-VLP antibody levels were 1:10 and 1:160 as measured by HI and ELISA tests, respectively.

Until now, the commercially available vaccines against RHDV have been produced from livers of infected rabbits. We describe here an attractive alternative to this method - a recombinant vaccine composed of empty capsids. The system of expression used by us - baculovirus/insect cells allows for high-level production of recombinant RHDV-VLP.

The VLPs obtained in this system visualized by electron microscopy were morphologically similar to native virons and were able to agglutinate human group 0 erythrocytes. After immunization, the recombinant particles induced RHDV-specific antibodies in rabbits and guinea pigs. Preliminary experiments show that rabbits immunized with VLPs were fully protected against challenge with virulent RHDV. This promising result indicates the potential of empty RHDV capsids as effective vaccination agents.

\section{Acknowledgements}

This work was supported by the State Committee for Scientific Research, grant 3P04B01923.

\section{REFERENCES}

Capucci L, Scicluna MT, Lavazza A (1991) Diagnosis of viral haemorrhagic disease of rabbits and the European brown hare syndrome. Rev Sci Tech 10: 347-370.

Capucci L, Frigoli G, Ronshold L, Lavazza A, Brocchi E, Rossi C (1995) Antigenicity of the rabbit hemorrhagic disease virus studied by its reactivity with monoclonal antibodies. Virus Res 37: 221-238.

Capucci L, Fallacara F, Grazioli S, Lavazza A, Pacciarini ML, Brocchi E (1998) A further step in the evolution of rabbit hemorrhagic disease virus: the appearance of the first consistent antigenic variant. Virus Res 58: 115-126.

Castanon S, Marin MS, Martin-Alonso JM, Boga A, Casais R, Humara JM, Ordas RJ, Parra F (1999) Immunization 
with potato plants expressing VP60 protein protects against rabbit hemorrhagic disease virus. J Virol 73: $4452-4455$.

Fitzner A, Kęsy A, Niedbalski W, Paprocka G (1999) Zastosowanie reakcji RT-PCR do identyfikacji szczepów wirusa krwotocznej choroby królików (RHD) wyosobnionych w Polsce. Medycyna Wet 55: 120-122 (in Polish).

Jiang X, Graham DY, Wang M, Estes MK (1992) Expression, self assembly and antigenicity of the Norwalk virus capsid protein. J Virol 66: 6527-6532.

Laemmli UK (1970) Cleavage of structural proteins during the assembly of the head of bacteriophage T4. Nature 227: 680-685.

Laurent S, Vautherot J-F, Madelaine M-F, Le Gall G, Rasschaert D (1994) Recombinant rabbit hemorrhagic disease virus capsid protein expressed in baculovirus self-assembles into viruslike particles and induces protecion. J Virol 68: 6794-6798.

Laurent S, Vautherot J-F, Gall le G, Madelaine M-F, Rasschaert D (1997) Structural, antigenic and immunogenic relationships between European brown hare syndrome virus and rabbit haemorrhagic disease virus. J Gen Virol 78: 2803-2811.

Le Gall G, Boilletot E, Morisse J-P (1992) Viral haemorrhagic disease of rabbit: purification and characterization of a strain isolated in France. Ann Rech Vet 23: 381387.

Liu SJ, Xue HP, Pu BQ, Quian NH (1984) A new viral disease in rabbits. Anim Husb Vet Med 16: 253-255.
Martinez-Torrecuadrada JL, Cortes E, Vela C, Langeveld JPM, Meloen RH, Dalsgaard K, Hamilton WDO, Casal JI (1998) Antigenic structure of the capsid protein of rabbit haemorrhagic disease virus. J Gen Virol 79: 19011909.

Meyers GC, Wirblich H, Thiel H-J (1991a) Rabbit hemorrhagic disease virus molecular-cloning and nucleotide sequencing of a calicivirus genome. Virology 184: 664676.

Meyers G, Wirblich CH, Thiel H-J (1991b) Genomic and subgenomic RNAs of rabbit hemorrhagic disease virus are both protein-linked and packaged into particles. $\mathrm{Vi}$ rology 184: 677-686.

Nagesha HS, Wang LF, Hyatt AD, Morrissy CJ, Lenghaus C, Westbury HA (1995) Self assembly, antigenicity, and immunogenicity of the rabbit haemorrhagic disease virus (Czechoslovakian strain V-351) capsid protein expressed in baculovirus. Arch Virol 140: 1095-1108.

Ohlinger VF, Haas B, Meyers G, Weiland F, Thiel H-J (1990) Identification of the virus causing rabbit hemorrhagic disease. J Virol 64: 3331-3336.

Parra F, Prieto M (1990) Purification and characterization of a calicivirus as the causative agent of a lethal hemorrhagic disease in rabbits. J Virol 64: 4013-4015.

Sibilia M, Boniotti MB, Angoscini P, Capucci L, Rossi C (1995) Two independent pathways of expression lead to self-assembly of the rabbit hemorrhagic disease virus capsid protein. J Virol 69: 5812-5815. 